\title{
DIGITALCOMMONS
} @WAYNESTATE-

Wayne State University

$11-22-2019$

\section{Finding Latent Groups in Observed Data: A Primer on Latent Profile Analysis in Mplus for Applied Researchers}

\author{
Sarah L. Ferguson \\ Rowan University \\ E. Whitney G. Moore \\ Wayne State University, whitneymoore@wayne.edu \\ Darrell M. Hull \\ University of North Texas
}

Follow this and additional works at: https://digitalcommons.wayne.edu/coe_khs

Part of the Education Commons, Kinesiology Commons, and the Sports Sciences Commons

\section{Recommended Citation}

Ferguson, S. L., Moore, E. W. G., \& Hull, D. M. (2020). Finding latent groups in observed data: A primer on latent profile analysis in Mplus for applied researchers. International Journal of Behavioral Development, 44(5), 458-468. DOI: 10.1177/0165025419881721

This Article is brought to you for free and open access by the College of Education at DigitalCommons@WayneState. It has been accepted for inclusion in Kinesiology, Health and Sport Studies by an authorized administrator of DigitalCommons@WayneState. 
International Journal of

Behavioral Development

88

\section{Finding Latent Groups in Observed Data: A Primer on Latent Profile Analysis in Mplus for Applied Researchers}

\begin{tabular}{|r|l|}
\hline Journal: & International Journal of Behavioral Development \\
\hline Manuscript ID & JBD-2019-05-3637.R1 \\
\hline Kanuscript Type: & Methods \& Measures \\
\hline Abstract: & $\begin{array}{l}\text { Keywords: } \\
\text { latent profile analysis, latent variable modeling, teaching paper } \\
\text { steps: (a) data inspection, (b) iterative evaluation of models, (c) model } \\
\text { fit and interpretability, (d) investigation of patterns of profiles in a } \\
\text { retained model, (e) covariate analysis, and (f) presentation of results. A } \\
\text { worked example is provided with syntax and results to exemplify the } \\
\text { steps. }\end{array}$ \\
\hline & $\begin{array}{l}\text { The present guide provides a practical guide to conducting latent profile } \\
\text { latily }\end{array}$ \\
\hline
\end{tabular}

\section{SCHOLARONE Manuscripts}


4

5

\begin{abstract}
The present guide provides a practical guide to conducting latent profile analysis (LPA) in the Mplus software system. This guide is intended for researchers familiar with some latent variable modeling but not LPA specifically. A general procedure for conducting LPA is provided in six steps: (a) data inspection, (b) iterative evaluation of models, (c) model fit and interpretability, (d) investigation of patterns of profiles in a retained model, (e) covariate analysis, and (f) presentation of results. A worked example is provided with syntax and results to exemplify the steps.
\end{abstract}

Keywords: latent profile analysis; latent variable modeling; teaching paper 
Finding Latent Groups in Observed Data: A Primer on Latent Profile Analysis in Mplus for Applied Researchers

The purpose of the present paper is to provide a practical guide on the use of latent profile analysis (LPA) in applied research studies. Use of LPA as an analysis approach has increased over recent years and is becoming more common in applied research. However, many applied researchers do not take formal coursework in advanced statistical analyses such as LPA (Henson, Hull, \& Williams, 2010), and may turn to the internet and research literature for assistance with teaching themselves new statistical techniques. Online resources and supports for LPA do exist (e.g. the Mplus manual and discussion boards, online lectures and class notes, etc.), but a unified guide for understanding and utilizing LPA with clear steps and examples specifically targeted for applied research does not currently appear in the literature. This paper is therefore intended for researchers already familiar with latent variable modeling, though not mixture modeling or LPA specifically. A general discussion of LPA will be presented followed by a worked example using data collected from a prior research study (Author, 2018). Syntax is also provided to conduct LPA in Mplus with a discussion of common options and additions for this program. The paper concludes with a discussion of LPA reporting practices, highlighting the primary information needed to report an LPA in applied research.

This work is intended predominately for applied researchers who are interested in exploring the applicability of LPA to their own research. Students still developing their methods and analysis expertise may also benefit from this introduction to LPA, as well as reviewers of research needing a brief introduction to the decision points and reporting practices of LPA. It must be noted that this is a primer on LPA and the foundational functions and processes of this analysis. As with any general introduction to a complex analysis, a balance is sought in this 
guide between clarity of LPA use in applied research contexts, and the complexity of potential issues with LPA analyses related to continuing debates in the field on key decisions (e.g. statistical power, covariate inclusion, etc.). Therefore, the more complex technical discussions and ongoing debates in the LPA and mixture modeling literature are outside of the scope of this work. This guide will focus on the practical decision points in LPA and suggest common approaches to these decisions supported by current methods research, while pointing the reader to additional sources to cover these issues in more depth.

\section{Latent Profile Analysis}

Latent profile analysis (LPA) and latent class analysis (LCA) are techniques for recovering hidden groups in data by obtaining the probability that individuals belong to different groups. This occurs through examination of the distributions of groups in the data and determining if those distributions are meaningful. It might be helpful to think of these groups, whether they are classes of people or profiles of people as unobserved latent mixture components. Indeed, both LCA and LPA are often referred to using the broad term Mixture Models. The distinction between LCA and LPA is the way that groups are defined on the basis of the observed variables. In LCA, observed variables are discrete, analogous to a binomial model (See Masyn, 2013 and Nylund-Gibson \& Choi, 2018 for more information). In LPA, observed variables are continuous, analogous to a gaussian model (Oberski, 2016). Additionally, there is latent transition analysis (LTA), which is any model that includes two or more latent class or profile constructs; these constructs can be informed by different indicators or the same indicators measured at different timepoints as a longitudinal extension (for a worked example, see NylundGibson, Grimm, Quirk, \& Furlong, 2014).

LPA, and the closely associated latent class analysis, are person-oriented approaches to 
latent variable analysis in the same family of methods as cluster analysis and mixture modeling (Bergman \& Magnusson, 1997; Bergman, Magnusson, \& El-Khouri, 2003; Collins \& Lanza, 2010; Gibson, 1959; Masyn, 2013; Sterba, 2013). Researchers new to LPA may benefit from a comparison to factor analysis methods, such as confirmatory factor analysis (CFA), as LPA also uses covariance matrices to explore relationships between observed data and latent variables (Bauer \& Curran, 2004; Bergman \& Magnusson, 1997; Bergman, et al., 2003; Marsh, Lüdtke, Trautwein, \& Morin, 2009; Masyn, 2013). However, where CFA uses a covariance matrix of items to uncover latent constructs, LPA uses a matrix of individuals to uncover latent groups of people. The main difference is "the common factor model decomposes the covariances to highlight relationships among variables, whereas the latent profile model decomposes the covariances to highlight relationships among individuals" (Bauer \& Curran, 2004, p. 6).

The person-oriented approach in LPA is grounded in three arguments. First, individual differences are present and important within an effect or phenomenon. Second, these differences occur in a logical way, which can be examined through patterns. Third, a small number of patterns (profiles in LPA) are meaningful and occur across individuals (Bergman \& Magnusson, 1997; Bergman, et al., 2003; Sterba, 2013). LPA is particularly useful for researchers in social sciences as patterns of shared behavior between and within samples may be missed when researchers conduct inter-individual, variable-centered analyses. For instance, if the LPA results in three profiles are relatively evenly spread across the sample, it is easy to miss the possibly meaningful differences between the profiles. LPA provides the opportunity to examine these profiles and what predicts or is predicted by membership within the different profiles. Variablecentered analyses assume the individuals within the sample all belong to a single profile or population with no differentiation between latent subgroups. 
LPA is undertaken in multiple steps, similar to structural equation modeling (SEM) analyses. In SEM, for instance, researchers will typically fit a conceptual model, check the measurement model to support their data, fit a series of structural models to identify the best fit of their data to their theoretical models (Bauer \& Curran, 2004; Kline, 2011). For LPA, the process is similar in that the researcher works through an iterative modeling process to identify the number of profiles to retain, fits a covariate model to explore the impact of these profiles on other variables in the study or predict profile membership (Masyn, 2013; Sterba, 2013). If the researcher also has a categorical grouping variable that they want to compare the profile structures across, they can conduct a multi-group LPA, including measurement invariance (Morin, Meyer, Creusier, \& Bietry, 2016). Researchers can also test for measurement invariance by covariate predictors by extending upon Masyn's (2017) description of measurement invariance testing for differential item functioning by covariates when conducting LCA models.

The overall goal of LPA is to uncover latent profiles or groups $(k)$ of individuals $(i)$ who share a meaningful and interpretable pattern of responses on the measures of interest $(j)$ (Bergman, et al., 2003; Marsh, et al., 2009; Masyn, 2013; Sterba, 2013). This is done using joint and marginal probabilities in within-class and between-class models. Two equations define the within-class model:

$$
\begin{aligned}
& y_{i j}=\mu_{j}^{(k)}+\varepsilon_{i j} \\
& \varepsilon_{i j} \sim N\left(0, \sigma_{j}^{2(k)}\right)
\end{aligned}
$$

where $\mu_{j}^{(k)}$ is the model implied mean and $\sigma_{j}^{2(k)}$ is the model implied variance, which will vary across $j=1 \ldots J$ outcomes and $k=1 \ldots K$ classes or profiles. The general assumptions of LPA include that outcome variables are normally distributed within each class and these within-class outcomes are locally independent (Sterba, 2013). The between-class model represents the 
probability of membership in a given class $k$ :

$$
p\left(c_{i}=k\right)=\exp \left(\omega^{(k)}\right) / \sum_{k=1}^{K} \exp \left(\omega^{(k)}\right)
$$

where $\omega^{(k)}$ is a multinomial intercept (fixed at 0 for the final class) and $c_{i}$ is the latent classification variable for the individual. The within-class and between-class models can therefore be combined into a single model using the law of total probability resulting in:

$$
f\left(\boldsymbol{y}_{i}\right)=\sum_{k=1}^{K} p\left(c_{i}=k\right) f\left(\boldsymbol{y}_{i} \mid c_{i}=k\right)
$$

which is the marginal probability density function for an individual $(i)$ after summing across the joint within-class density probabilities for the $J$ outcome variables, weighted by the probability of class or profile membership from equation 3. Finally, the LPA analysis results in a posterior probability for each individual defined as:

$$
t_{i k}=p\left(c_{i}=k \mid \boldsymbol{y}_{i}\right)=\frac{p\left(c_{i}=k\right) f\left(\boldsymbol{y}_{i} \mid c_{i}=k\right)}{f \boldsymbol{y}_{i}}
$$

representing the probability of an individual $(i)$ being assigned membership $\left(c_{i}\right)$ in a specific class or profile $(k)$ given their scores on the outcome variables in the $\boldsymbol{y}_{i}$ vector. A posterior probability $(t)$ is calculated for each individual in each profile, with values closer to 1.0 indicating a higher probability of membership in a specific profile. The more distinction between the posterior probabilities for an individual, the more certainty there is around their membership assignment (Sterba, 2013).

\section{Model Retention Decisions}

As LPA is a model testing process, multiple models are fit with varying levels of classes or profiles. The number of models to test depends on the research topic; often published LPA studies have found the best fitting model theoretically and statistically after comparing five to six models (Masyn, 2013; Tein, Coxe, \& Cham, 2013). Each model is then compared against the previous model or models to make a decision regarding the number of latent profiles in the data 
(Christie \& Masyn, 2008; Marsh, et al., 2009; Masyn, 2013). Commonly, decisions regarding model retention in LPA use Bayesian Information Criterion (BIC), Sample-Adjusted BIC (SABIC), and Akaike's Information Criterion (AIC) (Celeux \& Soromenho, 1996; Marsh, et al., 2009; Masyn, 2013; Tein, et al., 2013). BIC is used for model selection decisions with a lower BIC value representing the preferred model:

$$
\operatorname{BIC}(K)=-2 L(K)+v(K) \ln n
$$

with $v(K)$ representing the number of parameters to be estimated in the model. The BIC can be conservative, but it prefers parsimony in a model and has been shown to outperform other indices with more continuous indicators (Morgan, 2014; Nylund, Asparouhov, \& Muthén, 2007). An alternative is the SABIC, which adjusts the formula to account for $n$ and is less punitive on the number of parameters in the model (Tein, et al., 2013). SABIC has been supported as the most accurate information criteria index in simulation studies, particularly with smaller samples and low class separation (Kim, 2014; Morgan, 2014):

$$
\operatorname{SABIC}(K)=-2 L(K)+v(K) \ln n *((n+2) / 24)
$$

Finally, AIC is an inconsistent model fit measure as it does not have strong parsimony constraints, leading to more variation in the AIC values between models. Like BIC, lower values of AIC indicate better model fit. AIC is calculated as:

$$
\operatorname{AIC}(K)=-2 L(K)+2 v(K)
$$

With BIC, SABIC, and AIC, it should be noted that while lower values indicate better fit, lower is relative (Masyn, 2013). Therefore, attention should be given to the magnitude of difference. Consider two very different models, both of which are being examined for goodness of fit by evaluating the relative improvement produced by a 2-class structure to a 3-class structure. If one observes a reduction in BIC values from 18,000 to 17,950 for Example A, and a 
reduction from 800 to 750 for Example B, the absolute difference of 50 points is not equivalent for both models. The relative difference from 18,000 to 17,950 is smaller than the relative difference of 800 to 750 , and therefore the change for Example A might suggest equivalency where the change for Example B might suggest a meaningful difference. Researchers need to take the context into consideration when evaluating change between models, as there is no rule on what level of change in fit values is considered "meaningful" across the board.

Entropy, a measure of classification uncertainty, is less common as a model retention index due to lack of support in simulation studies (Masyn, 2013; Tein, et al., 2013). However, entropy as a statistical measure of uncertainty can still be useful in supporting LPA model retention as high entropy may indicate more classification uncertainty. Entropy is calculated as:

$$
\mathrm{E}(K)=\sum_{k=1}^{K} \sum_{i=1}^{n} t_{i k} \ln t_{i k}
$$

where $t_{i k}$ represents the posterior probabilities as shown in equation 5 . Entropy is, therefore, a measure of how well each LPA model partitions the data into profiles (Celeux \& Soromenho, 1996). Entropy can range from 0 to 1 with higher values representing better fit of the profiles to the data (Tein, et al., 2013). Note that this interpretation is somewhat counter-intuitive, as lower entropy values actually represent more uncertainty or chaos in the model, which is akin to saying lower values on the entropy statistic indicate more entropy (i.e. more classification uncertainty). Values of .80 or greater provide supporting evidence that profile classification of individuals in the model occurs with minimal uncertainty (Celeux \& Soromenho, 1996; Tein, et al., 2013).

Additionally, the Lo, Mendell, and Rubin (LMR) test is sometimes used to compare models, in a similar fashion to the $\chi^{2}$ difference test in other model testing analyses (Lo, Mendell, \& Rubin, 2001; Marsh, et al., 2009; Masyn, 2013; Tein, et al., 2013). LMR tests the likelihood ratio of one model as compared to another with an adjusted asymptotic distribution instead of a 
$\chi^{2}$ distribution (Lo, et al., 2001). As with the chi-square difference test, the LMR test is assessed for significance across difference in degrees of freedom. It is interpreted with a significance test in which statistical significance indicates the more parsimonious model (fewer profiles) represents the relationships present in the data significantly worse than the less parsimonious model (more profiles). In other words, the LMR test assists in determining when additional profiles are not improving fit or discrimination of the model. Thus, a non-significant LMR test suggests that the more parsimonious model is the better fitting and representative model.

Alternatively, the bootstrap likelihood ratio test (BLRT) can be used to evaluate the fit of one model compared to a model with one less profile $(k-1)$. BLRT uses parameter estimation methods to create multiple bootstrap samples to represent the sampling distribution (Masyn, 2013; McLachlan, 1987). A statistically significant BLRT indicates the current model is a better fit than a model with $k-1$ profiles. This approach has shown favorable results in simulation studies over the LMR test (Nylund, et al., 2007). However, both the LMR and the BLRT can suffer from never reaching a non-significant value, as the addition of parameters can represent more of the information contained within the data. In such a situation, it is recommended that the log likelihood values be plotted and examined for a bend or "elbow" to determine where the model improvement gain starts to diminish relative to the additional parameters estimated (Masyn, 2013).

Finally, as in any model testing analysis, theoretical support should exist for the final model retained, and patterns and profiles uncovered should be interpretable (Marsh, Hau, \& Wen, 2004; Marsh, et al., 2009; Masyn, 2013). Reliance upon theory and prior work to evaluate the reasonableness of a model is essential to LPA to ensure the final model and underlying profiles represent interpretable and meaningful groupings of individuals within the context of 
prior research. While it may be possible to produce models with more profiles that produce better fit, if this results in reduced distinction between profiles, ability to define and interpret those profiles, or increases the likelihood of capturing on chance or common error variance (e.g., method variance) rather than true classification distinctions, then the model with more profiles is not beneficial to theory, science, or practical application. Researchers should examine the underlying profiles produced in the retained model, including examination of trend or pattern lines of profiles, to determine when profiles may be near to one another in patterns such that distinctness is not meaningful. Profiles or classes containing less than $5 \%$ of the sample may be spurious, and the relevance of such profiles should be carefully considered and examined for interpretability and substantiveness (Marsh, et al, 2009; Masyn, 2013). Empirically, the lack of support for a small proportion profile may come from examining the results of the $k+1$ model to see if that profile "collapsed" or no longer appears in the results (Masyn, 2013). Lastly, the number of individuals represented by a small proportion profile should be taken into account, as a small percentage from a large sample may include sufficient individuals $(n=30-60)$ to support generalizability (Vincent \& Weir, 2012).

It is worth noting that good practice includes reporting different close fitting models and providing a detailed explanation of the decision-making process in selecting the retained model. This detail helps those attempting to replicate the existence of established profiles since the rational and decision-making process should be re-applied when possible. For example, if prior work suggested five profiles, but the fifth was removed as a spurious minor grouping, perhaps subsequent examinations that reveal a fifth profile would lead to the conclusion that it was not spurious after all. Alternatively, good fit for only four profiles would help to confirm prior judgment about the removal of the spurious, and non-theory fitting fifth profile. Additionally, 
class separation can be used to assist researchers in understanding the differences between the retained profiles. A profile plot of class probabilities is one way to evaluate profile separation, and odds ratios can be used to further evaluate the differences between profiles (Masyn, 2013).

Power and sample size requirements should also be considered for planned LPA studies. Power analysis in LPA is a developing area in the field with promising advances (Gudicha, 2015; Park \& Yu, 2017; Tein, et al., 2013). However, there is currently no simple formula or calculator to estimate required sample size in LPA. The required sample size is dependent on the number of profiles and the distance between the profiles, which is unknown in advance and can only be estimated based on prior research (Tein, et al., 2013). Across 38 studies, the median sample size was found to be $n=377$, while some simulation studies have suggested samples of 300 to 500 would qualify as a minimum sample (Finch \& Bronk, 2011; Nylund, et al., 2007; Peugh \& Fan, 2014; Tein, et al., 2013). Readers are referred to Preacher and Coffman (2006) and MacCallum, Browne, and Sugawara (1996) for further information on power analysis in covariance modeling.

\section{Covariate Analyses}

Following the profile retention decision in LPA is the examination of covariates to discover relationships and differences between latent groups (Clark \& Muthen, 2009; Marsh, et al., 2009; Nylund-Gibson \& Masyn, 2016). Exploring relationships with covariates provides additional information on the latent profiles and how the covariate variables may have differing effects on these profiles. One key point here, as highlighted in Marsh, et al. (2009), is the "covariates are assumed to be strictly antecedent variables" (p. 195) and have no effect on the formation of the profiles themselves. Historically, covariates were considered antecedents and not outcomes, because if the covariates were included in the model as outcomes, then they would alter the definition of the latent classes when entered into the model (Nylund-Gibson \& Masyn, 
2016). Thus, latent classes were regressed on the covariates of interest, not the other way around; which allowed for some freedom in the method of covariate inclusion, as these covariates should not influence the LPA profile solution. When to include covariates has been debated within the LPA/LCA literature; simulation studies have supported the preference for including covariates after the original model retention decision is made (Nylund-Gibson \& Masyn, 2016).

There are three methods of covariate inclusion in the current LPA literature. Method one is to examine the profiles based on each individual's most likely membership, and then explore the relationship of the profiles with the covariates in a separate post hoc analysis. Using this method, researchers first assign each individual to the profile for which they have the highest posterior probability. Then, the relationship between profile groups, as defined by individuals placed into their most likely profile, and the study covariates are evaluated. This turns the latent profiles into a categorical grouping variable, which decreases the computational burden of other approaches. However, this option is only recommended if entropy, or the level of classification uncertainty in the model, is above 0.80 (Clark \& Muthen, 2009). This is recommended because profile classifications are treated as fixed, categorical variables, rather than latent profiles with flexibility (e.g., the probability associated with them). If the entropy value is less than .80 (i.e., classification uncertainty in the model is increased), this approach may falsely force individuals into profiles without clear justification. Marsh, et al. (2009) and Christie and Masyn (2010) provide examples of this post hoc approach to covariate analysis.

Method two is a more advanced approach to LPA covariate inclusion called the ML three-step approach, which is also sometimes referred to as the VAM approach (Asparouhov \& Muthén, 2014; Vermunt, 2010). This approach follows the same first step as method one in that the latent profiles are evaluated first. In step two, the individuals are assigned to their most likely 
profile using the posterior probabilities provided from the initial latent profile analysis, similar to method one. However, the ML three-step approach approaches differs in the estimation of the model with the covariates included, because the profile assignment uncertainty is added into the model syntax by using the estimated average classification errors for each profile from step two. In this way, the third method accounts for the average uncertainty in profile assignment while modeling the effects of covariates on or with the profiles (Asparouhov \& Muthén, 2014; Vermunt, 2010). For further reading on this method, readers are pointed to Asparouhov and Muthén (2018) and Nylund-Gibson, Grimm, and Masyn (2018).

Finally, method three is the $\mathrm{BCH}$ approach, also a three-step approach (Asparouhov \& Muthen, 2018; Masyn, 2013; McLarnon \& O’Neill, 2018). The first step of the BCH approach is again determining the number of latent profiles without including the covariates in the model (Clark \& Muthen, 2009; Marsh, et al., 2009). Similar to the VAM approach described above, in the second step the participants' individual class probabilities are used to specify their probability of membership into each latent profile. Therefore, this method includes individual rather than average uncertainty in profile classification. Although this makes the $\mathrm{BCH}$ approach computationally complex, simulation studies have found this approach to be a relatively robust method over method one (Clark \& Muthen, 2009; Nylund-Gibson \& Masyn, 2016). There are now default commands available within Mplus for the most basic implementation of the $\mathrm{BCH}$ approach (see syntax for example of how to implement these steps; for further details regarding the limitations of the default command options in Mplus see Asparouhov \& Muthen, 2018). Using the $\mathrm{BCH}$ approach means that indicators for the profiles are present in the model with the covariates during analysis as shown in Figure 1. Note in the figure how the latent profiles predict responses on the indicators, while the antecedent covariates are predicting the profiles. The 
example provided in the current article uses this approach.

The second and third methods described above are currently recommended over the post hoc approach because they both allow for the uncertainty of profile assignment to remain in the model while also usually being able to maintain the integrity of the profiles when the covariates are added in. It is important to confirm that the profiles have not changed once the covariates are added into the model, as such fluctuations may indicate the presence of differential item functioning for one or more of the profile indicators in relation to the covariates (Masyn, 2017; Nylund-Gibson, Grimm, \& Masyn, 2019; Nylund-Gibson \& Masyn, 2016; Osterlind \& Everson, 2009). Great new work is being done to address this, which, to date, has shown it is possible to implement measurement invariance steps with predictor covariates that result in differential item functioning information regarding the indicators, profiles, and covariates. To learn more about this approach see Masyn (2017) and Osterlind and Everson (2009). Syntax examples for each of these approaches are provided in the supplemental files for this article.

\section{INSERT FIGURE 1 ABOUT HERE}

\section{Worked Example}

A worked example of the use of LPA with observed data is provided to walk the reader through the LPA process. Data for this example comes from a larger empirical study published elsewhere (see Author, 2018). The data is used here only as an example to demonstrate LPA, as opposed to the theoretical implications of the results. Data was collected from 295 high students from grades 11 and 12 in one public school district in the southern United States.

LPA was used as the primary inferential analysis method for the study. All latent profile analyses were conducted using Mplus 8 (Muthén \& Muthén, 1998-2017) with maximum likelihood estimation. LPA was used in two applications: first in exploring latent profiles of 
science occupational preference, and second to examine group differences in the resulting latent profiles based on study covariates. The research questions used to inform the study were:

1. Based on measures of science motivation, science attitude, science interest, and science achievement, how are latent profiles defined to represent occupational preferences for science career choice?

2. What is the magnitude of group differences in occupational preferences for science by sex, socio-economic status, personality, and cognitive ability?

\section{Steps of LPA}

There are six general steps in the LPA process we will detail in this example (see Figure 3). Step one, as with all analyses, the data should be cleaned for analysis and checked for standard statistical assumptions (e.g., normality of continuous variables, independence of observations, etc.; see Osborne, 2012). In this example, item-level missing values were estimated prior to the analysis process using maximum likelihood estimation in Mplus. Cases where all items were missing for one composite variable (i.e., missingness at the composite level) were estimated in the LPA process in Mplus utilizing full-information maximum likelihood (FIML). Participants were removed from the analysis if values on all variables in the study were missing $(n=4)$. As in other latent variable analyses, missing data can be handled by FIML or multiple imputation, depending on what is best for the scenario. Multiple imputation is recommended when a large dataset is being utilized to answer different research questions, planned missing data designs were implemented, or the computational burden for model convergence will be lessened utilizing imputed datasets rather than maximum likelihood estimation to handle the missingness while estimating the model (Baraldi \& Enders, 2010). In this example, we will highlight how to utilize FIML with Mplus, which can often address individuals' needs with 
smaller datasets and the use of informative auxiliary variables (Baraldi \& Enders, 2010; Howard, Rhemtulla, \& Little, 2015). Readers are referred to Osborne (2012) and Chapter 11 of the Mplus user's manual (Muthén \& Muthén, 1998-2017) for further introduction to missing data issues. Also of note in the present study is the use of composite variables as opposed to item-level data as indicators in the model for simplicity (i.e., reduce complexity and increase model convergence).

Step two involves evaluating a series of hypothetically plausible iterative LPA models, starting with a model with one profile and typically ending with a model estimating five or six profiles (Masyn, 2013; Tein, et al., 2013). Mplus syntax examples are provided in the Mplus Version 8 user's manual examples 7.9 and 7.12 (Muthén \& Muthén, 1998-2017). Samples of the basic LPA syntax can be found in Appendix A, and the syntax from this article's example may be found in Appendix B. In Mplus syntax, LPA is defined in two ways. First, the CLASSES command is added after the USEVARIABLES command to specify the number of classes or profiles being estimated in the model. So for the first one-profile model, CLASSES is set to c (1) as shown in the example. For a two-profile model this would be CLASSES = c (2), and so on. Second, the analysis TYPE is entered as MIXTURE, as LPA is a special type of mixture model. Additionally, Mplus output options can be requested to support the LPA analysis process (Muthén \& Muthén, 1998-2017). TECH 1 is used for multiple analyses in Mplus as it provides parameter specification and starting values for all estimated parameters in the model. This output can be helpful in identifying estimation errors. TECH 8 provides the optimization history for RANDOM, MIXTURE, and TWOLEVEL analyses in Mplus. TECH 11 is used to call for the LMR test to compare the current model against the prior model estimated with $k$ - 1 classes or profiles. Finally, TECH 14 calls for the bootstrap likelihood ratio test. Note that TECH 11 and 
TECH 14 cannot be provided if there is only one class or profile in the model, so this option must be removed from the syntax for the first model where only one profile is estimated (it is provided in the one-profile example here for reference only). Additionally, in the SAVE line, CPROBABILITIES is added to call for the class or profile probability estimates. This option provides the posterior probabilities for each individual in each latent class or profile as well as the most likely class membership for each individual in the sample. Also, note it is necessary to change the SAVEDATA file name for each model run to avoid overwriting existing SAVEDATA output files.

There are two other commands that are often used when conducting LPA that can assist in obtaining a solution in Mplus. The first is using the STARTS subcommand under ANALYSIS to adjust upward the number of either the initial stage starts in the maximization step and the final stage optimizations in the likelihood step of the ML estimation (Muthén \& Muthén, 19982017). This helps the iteration process of the ML or MLR estimators reach convergence by extending the number of attempts (Muthén \& Muthén, 1998-2017). It is recommended that the second value for the final stage optimizations be no more than a quarter of the initial stage starts value (Muthén, 2010; Muthén \& Muthén, 1998-2017).

Another approach that can assist in obtaining a solution for a model is providing start values. There are two ways to specify start values. One is to manually specify start values, which is done in the syntax by adding an asterisk (*) after a parameter in the model followed by the start value. For example, Y ON X*.50 instructs Mplus to use .50 as the start value for the regression coefficient Y ON X (i.e., X predicting Y). Another option, particularly if you have attempted to model a solution that did not converge, is to use the values the model estimation did reach for the parameter estimates as start values. Mplus always provides these start values in 
TECH 1 output, SVALUES, and, when the model does not converge, under the MODEL RESULTS (Muthén \& Muthén, 1998-2017). TECH1 output start values is a good section to examine if a particular parameter seems to be nearing an out of bounds estimation that results in non-convergence (e.g., correlation too close to 1). The SVALUES section will provide the model syntax including starting values based upon the model-estimated parameter values. This syntax can be copied and pasted into the input syntax file under the MODEL command to inform estimation in Mplus. As with TECH1, SVALUES can be included in the OUTPUT command line of any model. When a model does not converge, the MODEL RESULTS output is formatted for Mplus syntax so that it can be copied and pasted under the MODEL command in the syntax file. To test if the model resulted from a local maxima in the estimation process, different, random start values can be used to run the model again (see Hipp \& Bauer, 2006 for further information). Providing start values essentially helps the ML estimation process by informing the Mplus estimator to pick-up where it left off, and hopefully reach model convergence.

In the example, a latent profile analysis was used for Research Question 1 to identify the presence of latent profiles on measures of science interest, motivation, attitude, and academic experiences. Model 1 was estimated with only one profile, Model 2 with two profiles, and so on to Model 5 with five profiles. Model fit statistics are provided in Table 1.

\section{INSERT TABLE 1 ABOUT HERE}

Step three involves evaluating models to identify model fit and interpretability. In the example presented, Model 4 was retained as the best model to fit the data based on the low loglikelihood value, AIC, BIC, and SABIC values, high entropy value, non-significant LMR test, the smallest class contained more than $5 \%$ of the sample, and the profiles were supported by theory. The loglikelihood value revealed relatively large decreases until the difference between 
Model 4 and 5. This is also true of the AIC and SABIC trend across models. BIC was marginally lower for Model 4 in comparison to Model 5. All four of these statistics support models 4 and 5 as the better models. Entropy for both models 4 and 5 is above .80 and nearly the same. The LMR and BLRT tests are significant for Model 4, which means the four-profile model is a better representation than the three-profile model. However, LMR is not significant for Model 5, which supports the more parsimonious Model 4 as a better fit than the less parsimonious Model 5. The smallest profile in Model 4 comprises 16\% $(n=44)$ of sample participants, whereas the smallest profile for Model 5 comprises $2 \%(n=6)$ of sample participants. When a small number of participants from the sample are represented in a profile, as in Model 5, it is difficult to be confident the profile represents a distinct grouping that might be generalizable to other samples. Finally, profiles from Model 5 did not align with theory as well as Model 4 profiles, which makes it difficult to justify and interpret (see Author, 2018).

In step four of the LPA, the retained model is interpreted by examining patterns of the profiles and weights of included variables in each profile. The four-profile model for this example is detailed in Table 2. The means and standard deviations of variables used to create the profiles are presented for each profile, and all were found to be statistically significant in the model. Note that standard deviations are the same as they are constrained in Mplus by default. The differences between the four latent groups are largely due to differences in interest, motivation, and attitude towards science, which aligns with the theoretical approach used in the study (see Figure 2).

It may be helpful in reporting LPA to provide names or labels for the profiles based on the observed differences in included variables. However, researchers should be cautious in providing names to avoid a naming fallacy, suggesting the label assigned to a profile is correct 
and clearly understood, or a reification error suggesting the label represents a real construct (Kline, 2011; Masyn, 2013). In the example, Profile 1 contains, on average, students with the lowest level of interest in science, a more negative attitude and low motivation towards science, and a low GPA, so was referred to as "Low Science Interest and GPA." Profile 2 contains those students with the highest interest in science, high attitude and motivation scores, and the highest average GPA, so was called "High Science Interest and GPA". Profile 3 students are low in terms of science interest, but more towards the middle in science attitude and motivation, with a GPA almost as high on average as seen in Profile 2. This profile was called "Low Science Interest, High GPA.” Finally, Profile 4 contains students who are somewhat interested in science, have mid-level attitudes and motivations towards science, and a GPA lower than Profile 3 but higher than Profile 1. This profile was referred to as "Medium Science Interest and GPA." These profile names are illustrations of selecting names that are clear in the description without overstating the profile or being cumbersome. It is important that when directional words are used in naming (e.g., low, high, negative, positive) that they are accurate of not only the relative relationship between the profiles in the sample but also of the absolute magnitude of the variables/profiles that they are describing. Carefulness with naming ensures accuracy and clarity when interpreting results and when generalizing results beyond the study sample.

\section{INSERT TABLE 2 ABOUT HERE}

\section{INSERT FIGURE 2 ABOUT HERE}

Next, in step five, conduct a covariate analysis. This step should be included when: a) the LPA analysis indicates there are profiles worth interpreting further, and b) there is a theoretical reason to evaluate the impact of the covariates on the profiles. To add the covariates into the model for concurrent analysis in Mplus, start with the syntax from step two. However, this time add the variable information for covariates to the NAMES and USEVARIABLES lines. 
Additionally, in the MODEL section of the syntax, specify the model relationships. For the particular example included here, the two-step approach to covariate inclusion was used in the original study (see Supplemental Files for Mplus syntax examples of all approaches). The syntax subcommand "\%OVERALL\%" informs Mplus that the following lines describe the overall model, as opposed to \%class label\%, which can be used to indicate an adjustment to classspecific portions of the model such as constraining profiles to be equal on indicators or estimating the variances of indicators across profiles (Muthén \& Muthén, 1998-2017). Covariates are entered into the model as predictors of c in the line "c ON X1 X2 X3".

For the second research question in the example, profiles identified in Research Question 1 are further analyzed to evaluate effects of theoretically identified covariates. Covariates of interest are: personality; cognitive ability, both verbal and spatial; sex; and socio-economic status, as defined by parent education level and occupation. Covariates are added by regressing the latent profile construct on the covariates (e.g., c ON X1 X2 X3). As the research is focused on adolescents who would like to pursue science occupations, Profile 2 (Highest Science Interest \& GPA) was used as the reference group. Comparisons of all profiles against a specific profile as a reference group are provided in the Mplus syntax by default, and the decision on which profile is used as reference can be arbitrary in an exploratory analysis, or strategic to address a specific research question as shown here. For this example, group difference values and statistical significance tests presented in the output are tests between each profile and Profile 2 based upon the research question of the study. The results of this covariate analysis are presented in Table 3. However, individuals could replicate this approach using more than one profile as the reference profile depending on their research question.

\section{INSERT TABLE 3 ABOUT HERE}


Based on this analysis, some covariates produce significant differences across profiles. Results suggest sex is statistically significantly different between Profile 2 (Highest Science Interest \& GPA) and Profile 4 (Medium Science Interest \& GPA), with more females represented in the high science interest profile. This difference represents a difference in membership by sex, not necessarily in measurement. If measurement across genders was of research interest or concern, then a two-group (i.e., male and female) model could have been run to test for measurement invariance (i.e., differences in parameter values by sex) of the latent profile construct. Vocabulary scores are also statistically significantly different between Profile 2 (Highest Science Interest \& GPA) and both Profile 1 (Lowest Science Interest \& GPA) and Profile 4 (Medium Science Interest \& GPA). Negative coefficients indicate the high science interest group tends to have higher vocabulary scores than the other two profiles. Finally, personality is shown to be statistically, significantly different for Profile 1 (Lowest Science Interest \& GPA) as compared to Profile 2 (Highest Science Interest \& GPA). A cross-tabulation demonstrates the difference is that significantly more of the students considered "Well-Adjusted" fall into the high science interest profile (see Author, 2018).

Step six is preparing the results for dissemination, the final step in the analysis. Presentation of an LPA should generally follow the same sequence as the analysis. First, detail data cleaning conducted, what assumptions are checked and the result, and how missing data are handled. Include information about the software used for analysis (e.g., Mplus 8) and estimation decisions made (e.g. changes to random starts, estimation method). Second, report steps taken to estimate LPA models. How many models were included in the analysis, and why? Did any of the models not converge? Finally, detail all decisions and problems that occurred in this step and how any problems were addressed. 
Third, report in a table all models that were estimated and the diversity of appropriate fit indices that informed the gestalt decision process. We recommend including the loglikelihood value, AIC, BIC, SABIC, LMR test, and BLRT. Preference in model retention should be given to SABIC, BIC, and BLRT, based on the simulation studies discussed previously, but model retention decisions can be strengthened with agreement between multiple pieces of information (Kim, 2014; Masyn, 2013; Morgan, 2014; Nylund, Asparouhov, \& Muthén, 2007). Entropy may also be reported, particularly to support the accuracy of assigning individuals to profiles for further analysis. Reporting the percentage of the sample that is found in the smallest profile can also be a useful metric to support model retention decisions. There are other fit indices and metrics that have been recommended, and these may be included as the researcher feels is appropriate and/or as is common in a particular field's literature (Marsh, et al., 2009; Masyn, 2013; Nylund, et al., 2007; Tein, et al., 2013; Vermunt \& Magidson, 2002). Overall, a holistic approach to reporting metrics should be used to support model retention decisions. Model retention decisions should be justified by the majority of the metrics and indices included, even if some do not suggest the same model (Kline, 2011; Marsh, et al., 2004; Masyn, 2013; Nylund, et al., 2007; Schreiber, Nora, Stage, Barlow, \& King, 2006).

Fourth, describe the profiles from the retained model. Pay attention to indicator variables that appear to best differentiate between profiles and highlight the significant differences for the reader. Tables and graphs can both be used to help the reader understand the relationships between the profiles and the indicators. Naming the profiles is not necessary, but can be a helpful way for readers to differentiate between groups and understand what indicators appear meaningful. As the investigator, guide the reader in interpreting the profiles and implications for differences observed. 
Fifth, if applicable, detail the covariate analysis used. If covariates were added to the LPA model and examined concurrently or in a three-step approach, report results of this analysis. If individuals were assigned to profiles for further analysis independent of the LPA, justify this process and clearly detail steps taken. How were individuals assigned to a profile? Were individuals not clearly assigned to one profile, and if so how was this handled?

\section{INSERT FIGURE 3 ABOUT HERE}

\section{Conclusion}

This article is intended to serve as a primer on the use of latent profile analysis (LPA) in Mplus. LPA can be a very useful approach in research focused on personal behaviors and characteristics as it uses person-level indicators to sort individuals into latent profiles based on shared response patterns. LPA can be used in any social science research context where individual differences and/or underlying patterns of shared behavior may be of interest. The example presented here is one such application, but many other possibilities exist for applications of LPA. LPA models of family functioning (Rybak, et al., 2017), employees' commitment mindsets (Morin, Meyer, Creusier, \& Bietry, 2016), adolescents' coping strategies Aldridge \& Roesch, 2008), and school climate (Eck, Johnson, Bettencourt, \& Johnson, 2017) are examples of other psychological and behavioral phenomena that have been examined.

However, researchers should also be thoughtful of the assumptions and limitations of LPA before using this method in their own work. LPA assumes the underlying latent profiles are continuous, as opposed to categorical as would be found in latent class analysis. Additionally, variables selected for inputs and covariates should be both theoretically supported as well as appropriate for LPA. Using advanced statistical methods for their own sake does not improve research practice, but using a technique like LPA when appropriate and theoretically justified can 
increase our understanding of phenomena and uncover previously unknown differences or latent groupings in data that may be meaningful to the field. This is not intended as an exhaustive discussion of all current issues in LPA literature, and researchers should continue to evaluate new approaches and improvements in LPA practice when undertaking a new analysis. However, this resource is intended to be a starting place with a clear guide and discussion on the use of LPA in applied research for novice researchers and reviewers new to this approach. 


\section{References}

Aldridge, A. A. \& Roesch, S. C. (2008). Developing coping typologies of minority adolescents: A latent profile analysis. Journal of Adolescence, 31, 499-517. doi: 10.1016/j.adolescence.2007.08.005

Asparouhov, T., \& Muthén, B. (2014). Auxiliary variables in mixture modeling: Three-step approaches using M plus. Structural Equation Modeling: A Multidisciplinary Journal, $21(3), 329-341$.

Author (2018)

Baraldi, A. N. \& Enders, C. K. (2010). An introduction to modern missing data analyses. Journal of School Psychology, 48, 5 - 37. doi :10.1016/j.jsp.2009.10.001

Bauer, D. J., \& Curran, P. J. (2004). The integration of continuous and discrete latent variable models: Potential problems and promising opportunities. Psychological Methods, 9, 3-29. doi: $10.1037 / 1082-989 X .9 .1 .3$

Bergman, L. R., \& Magnusson, D. (1997). A person-oriented approach in research on developmental psychopathology. Development and Psychopathology, 9, 291-319. doi: $10.1017 / \mathrm{S} 095457949700206 \mathrm{X}$

Bergman, L. R., Magnusson, D., \& El Khouri, B. M. (2003). Studying individual development in an interindividual context: A person-oriented approach. Mahwah, NJ: Psychology Press.

Celeux, G., \& Soromenho, G. (1996). An entropy criterion for assessing the number of clusters in a mixture model. Journal of Classification, 13, 195-212.

Clark, S. L., \& Muthén, B. (2009). Relating latent class analysis results to variables not included in the analysis. Available online: http://hbanaszak.mjr.uw.edu.pl/TempTxt/relatinglca.pdf

Collins, L. M., \& Lanza, S. T. (2013). Latent class and latent transition analysis: With 
FINDING LATENT GROUPS

applications in the social, behavioral, and health sciences. Hoboken, NJ: John Wiley \& Sons.

Christie, C. A., \& Masyn, K. E. (2008). Latent profiles of evaluators' self-reported practices. The Canadian Journal of Program Evaluation, 23(2), 225-254.

Eck, K. V., Johnson, S. R., Bettencourt, A., \& Johnson, S. L. (2017). How school climate relates to chronic absence: A multi-level latent profile analysis. Journal of School Psychology, 61, 89-102. doi: 10.1016/j.jsp.2016.10.001

Gibson, W. A. (1959). Three multivariate models: Factor analysis, latent structure analysis, and latent profile analysis. Psychometrika, 24, 229-252.

Gudicha, D. (2015). Power analysis methods for tests in latent class and latent Markov models Ridderkerk, The Netherlands: Ridderprint BV.

Henson, R. K., Hull, D. M., \& Williams, C. S. (2010). Methodology in our education research culture: Toward a stronger collective quantitative proficiency. Educational Researcher, 39(3), 229-240.

Howard, W. J., Rhemtulla, M., \& Little, T. D. (2015). Using principal components as auxiliary variables in missing data estimation. Multivariate Behavioral Research, 50(3), 285-299.

Kim, S. Y. (2014). Determining the number of latent classes in single-and multiphase growth mixture models. Structural Equation Modeling, 21(2), 263-279. doi:

$10.1080 / 10705511.2014 .882690$

Kline, R. B. (2011). Principles and practice of structural equation modeling (3rd Ed.). New York, NY: Guilford Press.

Lo, Y., Mendell, N. R., \& Rubin, D. B. (2001). Testing the number of components in a normal mixture. Biometrika, 88, 767-778. 
MacCallum, R. C., Browne, M. W., \& Sugawara, H. M. (1996). Power analysis and determination of sample size for covariance structure modeling. Psychological methods, 1(2), 130-149.

Marsh, H. W., Hau, K. T., \& Wen, Z. (2004). In search of golden rules: Comment on hypothesistesting approaches to setting cutoff values for fit indexes and dangers in overgeneralizing Hu and Bentler's (1999) findings. Structural equation modeling, 11, 320-341. doi: $10.1207 / \mathrm{s} 15328007 \mathrm{sem} 1103 \_2$

Marsh, H. W., Lüdtke, O., Trautwein, U., \& Morin, A. J. (2009). Classical latent profile analysis of academic self-concept dimensions: Synergy of person-and variable-centered approaches to theoretical models of self-concept. Structural Equation Modeling, 16, 191-225. doi:

$10.1080 / 10705510902751010$

Masyn, K. E. (2013). Latent class analysis and finite mixture modeling. In T. Little (Eds), The Oxford Handbook of Quantitative Methods (551-611). New York, NY: Oxford University Press.

Masyn, K. E. (2017). Measurement invariance and differential item functioning in latent class analysis with stepwise multiple indicator multiple cause modeling. Structural Equation Modeling: A Multidisciplinary Journal, 24(2), 180-197.

McLachlan, G. J. (1987). On bootstrapping the likelihood ratio test statistic for the number of components in a normal mixture. Applied Statistics, 36, 318-324. doi: 10.2307/2347790

McLarnon, M. J. W. \& O’Neill, T. A. (2018). Extensions of auxiliary variable approaches for the investigation of mediation, moderation, and conditional effects in mixture models. Organizational Research Methods, 21(4), 955-982. doi: 10.1177/1094428118770731.

Morgan, G. B. (2015). Mixed mode latent class analysis: An examination of fit index 
performance for classification. Structural Equation Modeling, 22(1), 76-86. doi: $10.1080 / 10705511.2014 .935751$

Morin, A. J. S., Meyer, J. P., Creusier, J., \& Bietry, F. (2016). Multiple-group analysis of similarity in latent profile solutions. Organizational Research Methods, 19(2), 231-254. doi: $10.1177 / 1094428115621148$

Muthén, L. (2010, November 29). Re: Latent profile analysis [Online discussion group]. Retrieved from http://www.statmodel.com/discussion/messages/13/115.html?1507757139

Muthén, L.K. and Muthén, B.O. (1998-2017). Mplus user's guide. (8th Ed.). Los Angeles, CA: Muthén \& Muthén

Nylund, K. L., Asparouhov, T., \& Muthén, B. O. (2007). Deciding on the number of classes in latent class analysis and growth mixture modeling: A Monte Carlo simulation study. Structural Equation Modeling, 14(4), 535-569. doi: 10.1080/10705510701575396

Nylund-Gibson, K. \& Choi, A. Y. (2018). Ten frequently asked questions about latent class analysis. Translational Issues in Psychological Sciences, 4(4), 440-461. doi:

\section{$10.1037 / \operatorname{tps} 0000176$}

Nylund-Gibson, K. \& Masyn, K. E. (2016). Covariates and mixture modeling: Results of a simulation study exploring the impact of misspecified effects on class enumeration. Structural Equation Modeling, 23, 782-797. doi: 10.1080/10705511.2016.1221313

Nylund-Gibson, K., Grimm, R., Quirk, M., \& Furlong, M. (2014). A latent transition mixture model using the three-step specification. Structural Equation Modeling: A Multidisciplinary Journal, 21, 1-16. doi: 10.1080/10705511.2014.915375

Oberski, D. (2016). Mixture models: Latent profile and latent class analysis. In J. Robertson and 
M. Kaptein (Eds.), Modern Statistical Methods for HCI. Springer International Publishing: Cham, Switzerland.

Osborne, J. W. (2012). Best practices in data cleaning: A complete guide to everything you need to do before and after collecting your data. Thousand Oaks, CA: Sage.

Osterlind, S. J., \& Everson, H. T. (2009). Differential item functioning (Vol. 161). Sage Publications.

Park, J., \& Yu, H. T. (2017). Recommendations on the sample sizes for multilevel latent class models. Educational and Psychological Measurement, Available online: http://journals.sagepub.com/doi/abs/10.1177/0013164417719111. doi: $10.1177 / 0013164417719111$.

Peugh, J., \& Fan, X. (2015). Enumeration index performance in generalized growth mixture models: A Monte Carlo test of Muthén's (2003) hypothesis. Structural Equation Modeling, 22(1), 115-131. doi: 10.1080/10705511.2014.919823

Preacher, K. J., \& Coffman, D. L. (2006, May). Computing power and minimum sample size for RMSEA [Computer software]. Available from http://quantpsy.org/.

Rybak, T. M., Ali, J. S., Berlin, K. S., Klages, K. L., Banks, G. G., Kamody, R. C., Ferry, R. J., Alemzadeh, R., \& Diaz-Thomas, A. M. (2017). Patterns of family functioning and diabetes-specific conflict in relation to glycemic control and health-related quality of life among youth with Type I Diabetes. Journal of Pediatric Psychology, 42(1), 40-51. doi: 10.1093/jpepsy/jsw071

Schreiber, J. B., Nora, A., Stage, F. K., Barlow, E. A., \& King, J. (2006). Reporting structural equation modeling and confirmatory factor analysis results: A review. Journal of Educational Research, 99, 323-338. 
Sterba, S. K. (2013). Understanding linkages among mixture models. Multivariate Behavioral Research, 48, 775-815. doi:10.1080/00273171.2013.827564

Tein, J. Y., Coxe, S., \& Cham, H. (2013). Statistical power to detect the correct number of classes in latent profile analysis. Structural Equation Modeling, 20, 640-657. doi: $10.1080 / 10705511.2013 .824781$

Vermunt, J. K. (2010). Latent class modeling with covariates: Two improved three-step approaches. Political Analysis, 18(4), 450-469.

Vermunt, J. K., \& Magidson, J. (2002). Latent class cluster analysis. In J. Hagenaars, \& A. McCutcheon (Eds.), Applied latent class analysis. (pp. 89-106). Cambridge: Cambridge University Press.

Vincent, W. J. \& Weir, J. P. (2012). Statistics in Kinesiology (4th Ed). Champaign, IL: Human Kinetics. 
FINDING LATENT GROUPS

Table 1

LPA Model Fit Summary for Research Question 1

\begin{tabular}{ccccccccccc}
\hline Model & $\begin{array}{c}\text { Log } \\
\text { likelihood }\end{array}$ & AIC & BIC & SABIC & Entropy $\begin{array}{c}\text { Smallest } \\
\text { Class } \%\end{array}$ & $\begin{array}{c}\text { LMR } \\
p \text {-value }\end{array}$ & $\begin{array}{c}\text { LMR } \\
\text { Meaning }\end{array}$ & $\begin{array}{c}\text { BLRT } \\
p \text {-value }\end{array}$ & $\begin{array}{c}\text { BLRT } \\
\text { Meaning }\end{array}$ \\
\hline 1 & -8909.94 & 17867.88 & 17955.96 & 17879.85 & & & - & - & - & - \\
2 & -8507.38 & 17088.75 & 17224.54 & 17107.21 & 0.89 & $45 \%$ & $<0.001$ & $2>1$ & $<0.001$ & $2>1$ \\
3 & -8370.30 & 16840.60 & 17024.10 & 16865.54 & 0.90 & $30 \%$ & 0.002 & $3>2$ & $<0.001$ & $3>2$ \\
4 & -8290.15 & 16706.31 & 16937.51 & 16737.73 & 0.89 & $16 \%$ & 0.010 & $4>3$ & $<0.001$ & $4>3$ \\
5 & -8253.86 & 16659.72 & 16938.63 & 16697.62 & 0.90 & $2 \%$ & 0.614 & $5<4$ & $<0.001$ & $5>4$ \\
\hline
\end{tabular}

Note. $n=295$, The Lo-Mendell Ruben (LMR) test and the bootstrap likelihood ratio test (BLRT) compare the current model to a model with $k-1$ profiles. 
FINDING LATENT GROUPS

Table 2

Four-Profile Model Results

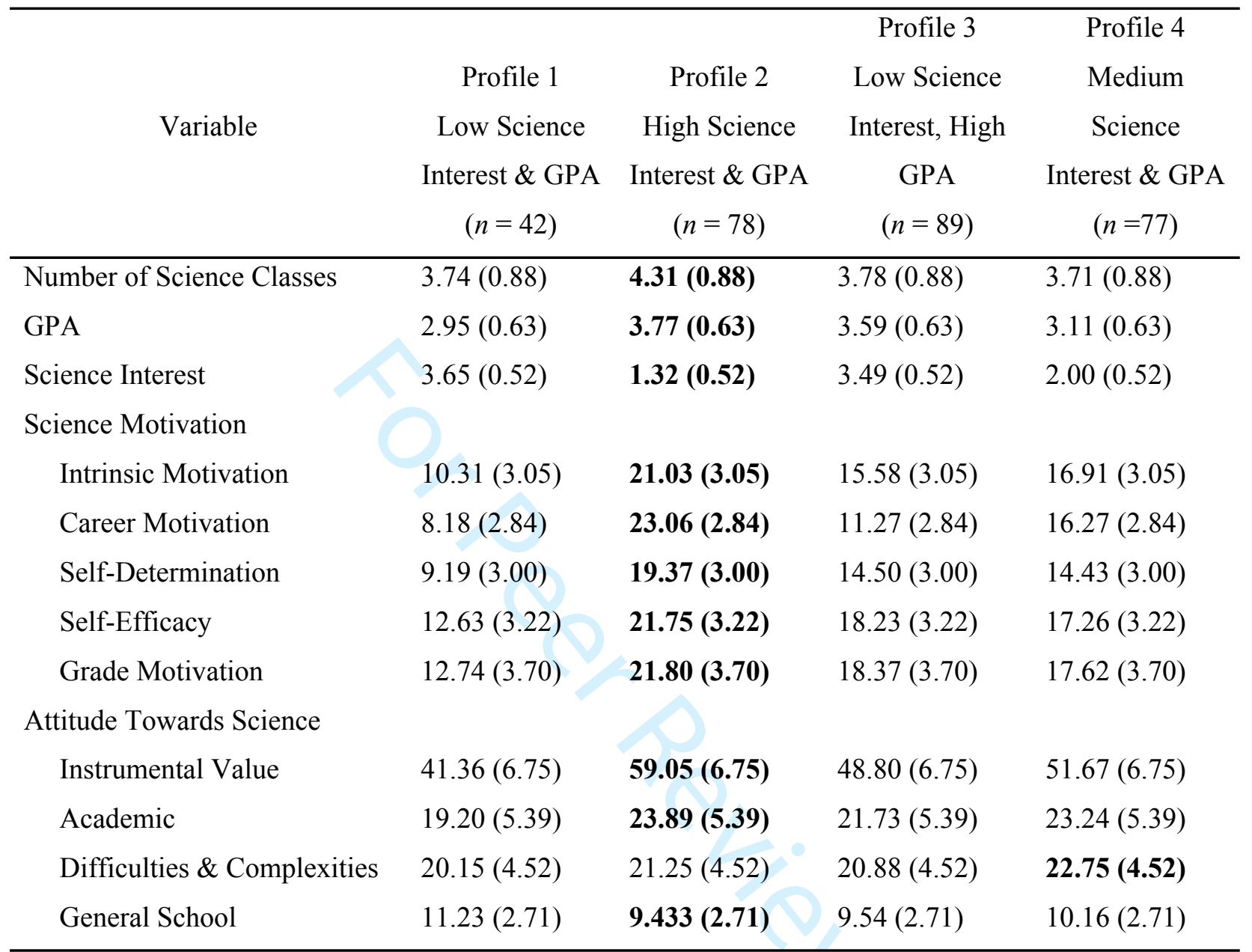

Note. Values respresenting highest positive response in bold (Science Interest and ATS-General School are reverse coded). Means and standard deviations for variables across all profiles: Number of Science Classes $M=3.78(S D=0.90)$, GPA $M=3.41(S D=0.70)$, Science Interest $M=2.00$ ( $\mathrm{SD}=0.50)$, Intrinsic Motivation $M=16.58(S D=4.60)$, Career Motivation $M=15.32(S D=6.11)$, Self-Determination $M=14.95$ ( $S D=4.42)$, Self-Efficacy $M=18.02(S D=4.35)$, Grade Motivation $M=18.20(S D=4.69)$, Instrumental Value $M=51.18(S D=8.90)$, Academic $M=22.34$ ( $S D=5.63)$, Difficulties \& Complexities $M=21.38$ $(S D=4.62)$, General School $M=9.94(S D=2.78)$ 
FINDING LATENT GROUPS

Table 3

Covariate Analysis Results for the 4-Profile Model

\begin{tabular}{lccc}
\hline \multicolumn{1}{c}{ Variable } & Profile 1 Low & Profile 3 Low & Profile 4 Medium \\
& Science Interest \& & Science Interest, & Science Interest \& \\
& GPA $(n=42)$ & High GPA $(n=89)$ & GPA $(n=77)$ \\
\hline Sex & -0.96 & -0.32 & $-1.373^{*}$ \\
Shipley-2 Vocabulary & $-0.19^{*}$ & -0.10 & $-0.23^{*}$ \\
Shiple-2 Block Patterns & -0.07 & -0.002 & -0.05 \\
Mother's SES & 0.02 & -0.02 & 0.003 \\
Father's SES & -0.02 & 0.01 & -0.002 \\
Personality & $-1.08^{*}$ & -0.12 & -0.556 \\
\hline
\end{tabular}

Note. ${ }^{*}=p<.05$, Profile 2 (High Science Interest \& GPA, $n=78$ ) served as reference group. 


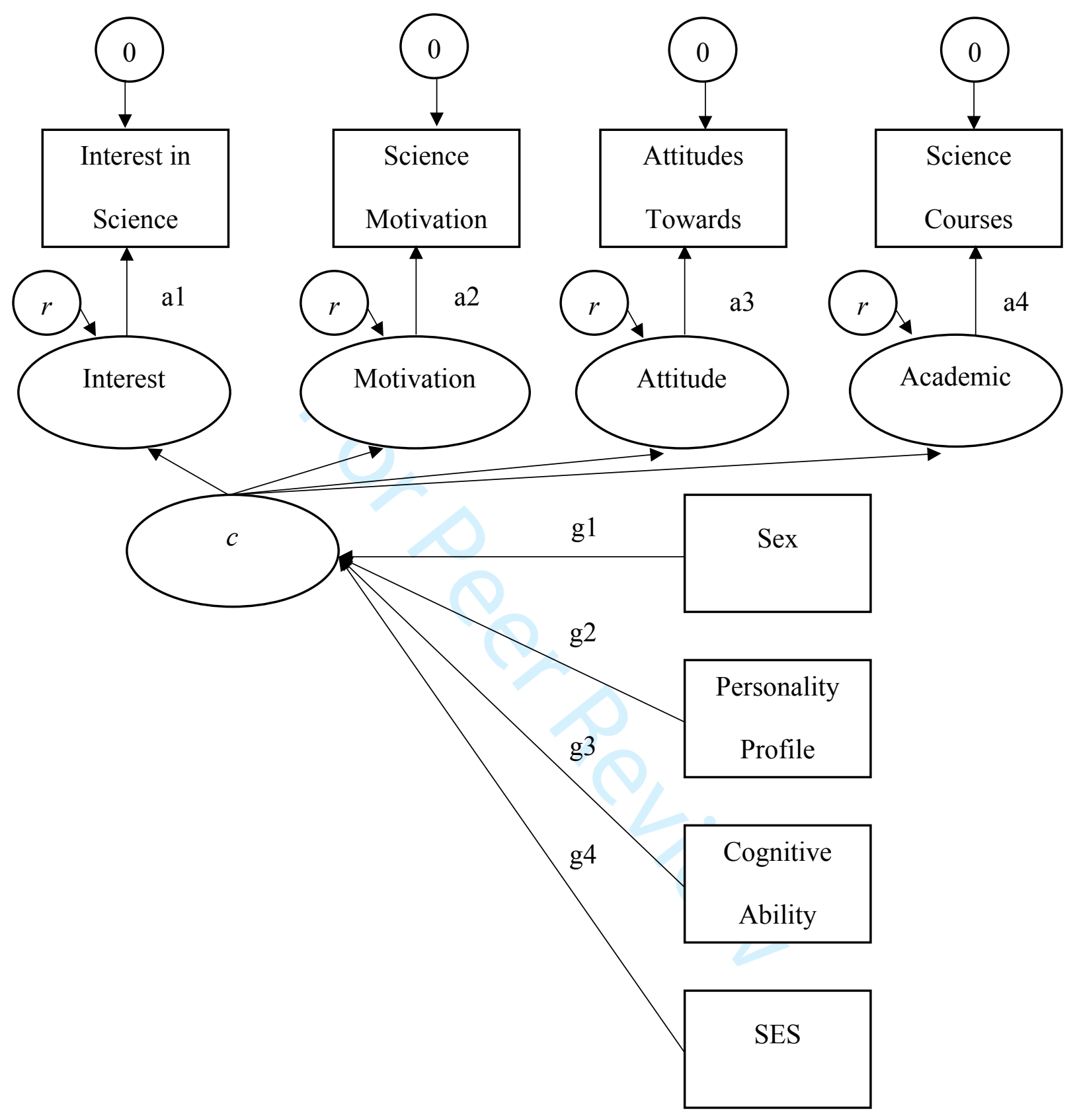

Figure 1. Example of LPA model with covariates 


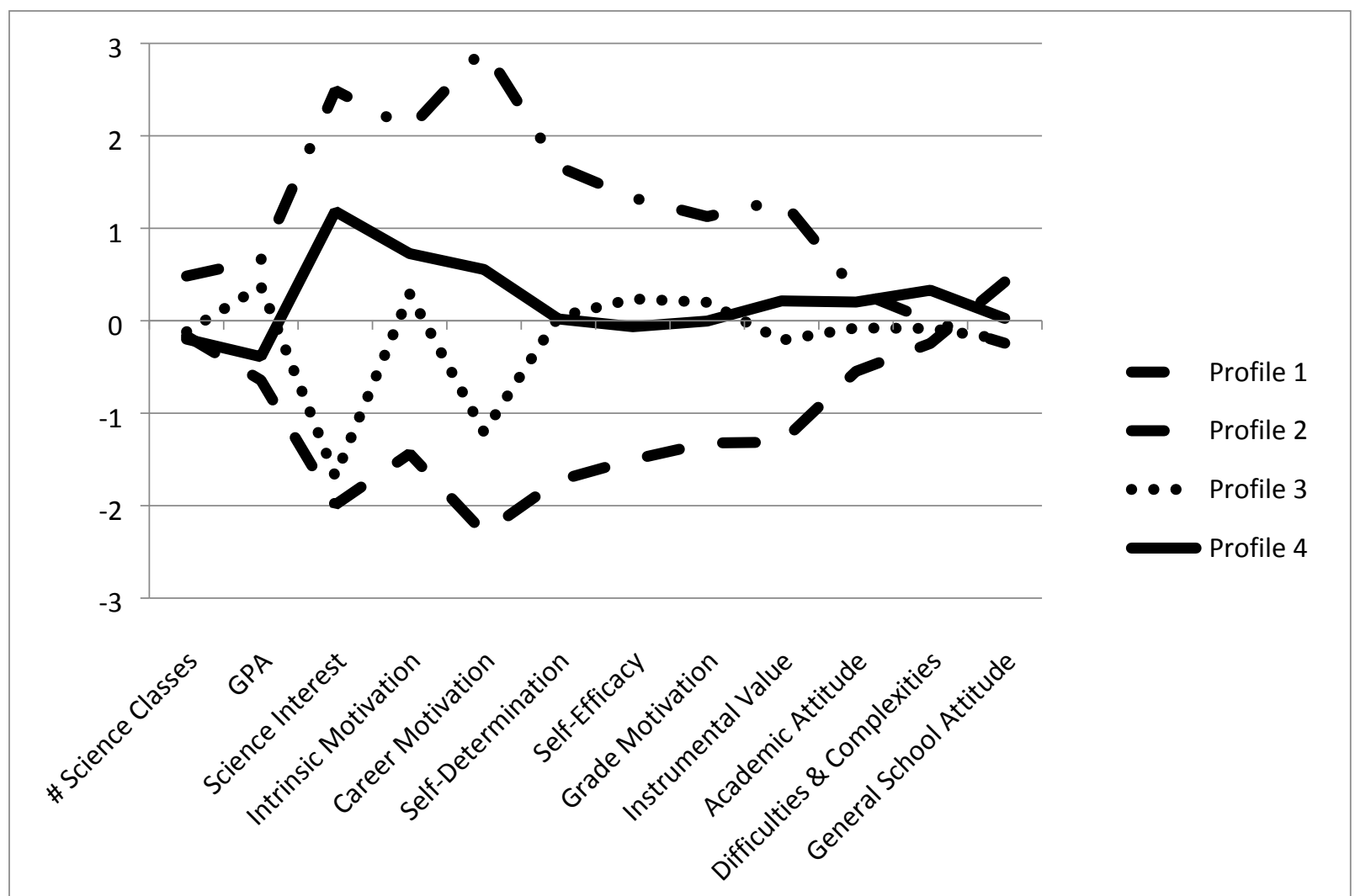

Figure 2. Line graph comparing profiles on indicator variables in z-score format

Note. Profile $1 n=42$; Profile $2 n=78$; Profile $3 n=89$; Profile $4 n=77$. 
FINDING LATENT GROUPS

Figure 3. Six foundational steps of latent profile analysis 


\section{Appendix A: Mplus Syntax Simple Example}

\section{One Profile LPA Model}

TITLE: $\quad$ LPA generic 2 profile syntax example

DATA:

FILE IS data.dat;

! Specifies file location for data file. Make sure data is in format appropriate for Mplus analysis

! per Mplus manual. This data file is in individual format (one row of data per participant)

VARIABLE:

NAMES ARE Y1-Y5 X1-X3;

! All variables included in data file should be named here.

!X1-X3 is shorthand for X1 through X3.

USEVARIABLES ARE Y1-Y5;

! Only variables intended for use in the analysis should be listed here

CLASSES = c (2);

! This is where you instruct Mplus on how many classes/profiles are being estimated. Initial

! model contains only one class/profile, thus it would be CLASSES = c (1). Above specifies two !profiles, and for each further iterative models the number in parentheses increases by one, so !three profiles/classes would be c (3), and so on.

MISSING ARE ;

! Used to communicate how missing data is coded in data file. Here shown with a "." which is ! all that is included in each cell with missing data in the data file

ANALYSIS:

TYPE = MIXTURE;

! LPA is a version of mixture modeling, and this instructs Mplus to analyze in this way

ESTIMATOR $=$ MLR;

!FIML robust to non-normal data

STARTS $=1000250$

STITERATIONS = 500;

! Default number of starts for each step of the ML estimation. First STARTS value specifies the !number of unique start values to start with, the 250 represents the 250 best unique start values !carrying forward to completion. The STITERATIONS specifies the number of ML iteration !steps for those 250 selected start values to go through to be able to converge. This is a! !maximum number of iteration; if a model converges in less than 500 iterations it will stop !before reaching 500 iterations.

!These values can be increased ... see "Four-Profile Final Model with Covariate Analysis !Syntax" for an example.

LRTSTARTS = 215010 ;

LRTBOOSTRAP $=250$; 
!The above start values are for the defaults for the LRT statistic being run to compare the current !model fit with the model fit of a model with one less class (k-1). The BOOTSTRAP statement !specifies the number of bootstrap draws to inform Mplus' bootstrapped LRT results.

MODEL:

!For a default Mplus model the LPA model does not need to be specified. However, it can be. !The model can also be modified from the Mplus default of estimating the indicator means (uniquely across profiles) and variances (constrained across profiles), as well as the latent !profile mean.

$\%$ OVERALL \%

[Y1-Y5]; !estimates the 5 indicators means for each profile. Without a label after the brackets, !the means are freely estimated in each profile, not constrained.

Y1-Y5 (Var1-Var5); !Label Var1-Var5 constrains the estimates of the variances across the !profiles to be equal.

OUTPUT:

TECH1 TECH8 TECH11 TECH14;

! TECH1 provides parameter specifications and starting values for the analysis

! TECH8 provides optimization history for this analysis type

!TECH11 provides LRT results

!TECH14 provides bootstrapped LRT test

SAVEDATA:

FILE IS LPA2.dat;

! Tells Mplus where to save the output files from the analysis

SAVE $=$ CPROBABILITIES;

! The above command lines are to save the most likely profile membership for each participant ! and the posterior probabilities for their membership in each latent profile. 


\section{LPA Model with Covariate Analysis}

TITLE: $\quad$ LPA generic syntax example with covariates

DATA:

FILE IS data.dat;

! Specifies file location for data file. Make sure data is in format appropriate for Mplus analysis ! per Mplus manual. This data file is in individual format (one row of data per participant)

VARIABLE:

NAMES ARE Y1-Y5 X1-X3;

! All variables included in data file should be named here

USEVARIABLES ARE Y1-Y5 X1-X3;

! All variables intended for use in the analysis should be listed here. The covariate variables are !included for this particular version of syntax though they were not included during the class

!enumeration steps.

CLASSES = c (4);

! This is where you instruct Mplus on how many classes/profiles are being estimated.

!For this version of covariate inclusion you would retain the best fitting model from prior !analyses without covariates

!In this example a model with 4 profiles/classes so CLASSES=c (4)

MISSING ARE ;

! Used to communicate how missing data is coded in data file, here shown with a "." and this is

! included in in each cell with missing data in the data file

ANALYSIS:

TYPE = MIXTURE;

! LPA is a version of mixture modeling, and this instructs Mplus to analyze in this way

!See above model ANALYSIS syntax for additional options that can be added to improve model !estimation and convergence.

MODEL:

$\%$ OVERALL $\%$

c ON X1 X2 X3;

! New inclusion here instructs Mplus to run the model as before and then add the covariates X1

! X2 and X3 as predictors of "c" classes/profiles

OUTPUT:

TECH1 TECH8 TECH11 TECH14;

! TECH1 provides parameter specifications and starting values for the analysis

! TECH8 provides optimization history for this analysis type

! TECH11 provides LMR test comparing this model to the previous model.

! (cannot be calculated with one profile model) 
! TECH14 provides BLRT comparing this model to the previous model.

! (cannot be calculated with one profile model)

SAVEDATA:

FILE IS LPA4Cov.dat;

! Tells Mplus where to save the output files from the analysis

SAVE $=$ CPROBABILITIES;

! The above command lines are to save the most likely profile membership for each participant ! and the posterior probabilities for their membership in each latent profile 


\title{
Appendix B: Mplus Syntax from Example Study
}

\section{Enumeration Step}

\section{Two-Profile LPA Syntax 4 Classes}

TITLE: Latent Profile Analysis Model 1, 2 Profile, Students

DATA:

FILE IS Mplus_LPA_RQ1_Final.csv;

! Specifies file location for data file. Make sure data is in format appropriate for Mplus analysis

! per Mplus manual. This data file is in individual format (one row of data per participant)

VARIABLE:

NAMES ARE ID Sex MomEd MomOcc DadEd

DadOcc ClassNum GPA Plans IPIP N IPIP E IPIP O IPIP A

IPIP_C SM_IM SM_CM SM_SD SM_SE - SM_GM $\overline{-}$ ATS_IV

ATS_AP ATS_DC ATS_GS Ship_V Ship_B - MomSES

DadSES;

! All variables included in data file should be named here.

USEVARIABLES ClassNum GPA Plans SM_IM SM_CM SM_SD SM_SE SM_GM ATS_IV ATS_AP ATS_DC ATS_GS;

! Selecting variables to use in the model (all profile indicators, no covariates at this stage)

CLASSES $=\mathrm{c}(4)$

! This is where you instruct Mplus on how many classes/profiles are being estimated. Initial

! model contains only one class/profile, thus it would be CLASSES = c (1). Above specifies four !profiles, and for each further iterative models the number in parentheses increases by one.

MISSING ARE ;

! Used to communicate how missing data is coded in data file. Here shown with a "." which is

! all that is included in each cell with missing data in the data file

\author{
ANALYSIS: \\ TYPE=MIXTURE; \\ ! LPA is a version of mixture modeling, and this instructs Mplus to analyze in this way \\ ESTIMATOR $=$ MLR; \\ !FIML robust to non-normal data \\ STARTS $=1000250$ \\ STITERATIONS $=500$; \\ ! Default number of starts for each step of the ML estimation. First STARTS value specifies the \\ !number of unique start values to start with, the 250 represents the 250 best unique start values \\ !carrying forward to completion. The STITERATIONS specifies the number of ML iteration
}


!steps for those 250 selected start values to go through to be able to converge. This is a! !maximum number of iteration; if a model converges in less than 500 iterations it will stop !before reaching 500 iterations.

!These values can be increased ... see "Four-Profile Final Model with Covariate Analysis !Syntax" for an example.

LRTSTARTS = 215010 ;

LRTBOOSTRAP = 250;

!The above start values are for the defaults for the LRT statistic being run to compare the current !model fit with the model fit of a model with one less class (k-1). The BOOTSTRAP statement !specifies the number of bootstrap draws to inform Mplus' bootstrapped LRT results.

\section{MODEL:}

!For a default Mplus model the LPA model does not need to be specified. However, it can be. !The model can also be modified from the Mplus default of estimating the indicator means !(uniquely across profiles) and variances (constrained across profiles), as well as the latent !profile mean. The syntax below specifies the Mplus defaults.

$\%$ OVERALL $\%$

[ClassNum GPA Plans SM_IM SM_CM SM_SD SM_SE SM_GM ATS_IV

ATS_AP ATS_DC ATS_GS]; !estimates the indicator means for each profile. Without a label !after the brackets, the means are freely estimated in each profile, not constrained.

ClassNum GPA Plans SM_IM SM_CM SM_SD SM_SE SM_GM ATS_IV ATS_AP ATS_DC ATS_GS (Var1-Var12); !Label Var1-Var12 constrains the estimates of the !variances across the profiles to be equal.

OUTPUT:

TECH1 TECH8 TECH11 TECH14;

! TECH1 provides parameter specifications and starting values for the analysis

! TECH8 provides optimization history for this analysis type

!TECH11 provides LRT results

!TECH14 provides bootstrapped LRT test

\section{SAVEDATA:}

FILE IS LPA1_2_FINAL.dat;

! Tells Mplus where to save the output files from the analysis

SAVE $=$ CPROBABILITIES;

! The above command lines are to save the most likely profile membership for each participant ! and the posterior probabilities for their membership in each latent profile. 


\section{Appendix C: Mplus Syntax from Example Study \\ Four-Profile Final Model with BCH Covariate Analysis Syntax}

Step 2, after enumeration phase completed is to create a file with the $\mathrm{BCH}$ weights and covariates in the same file.

TITLE: Latent Profile Analysis Model 1, 4 Profile, Students

DATA:

FILE IS Mplus_LPA_RQ1_Final.csv;

VARIABLE:

NAMES ARE ID Sex MomEd MomOcc DadEd

DadOcc ClassNum GPA Plans IPIP_N IPIP_E IPIP_O IPIP_A

IPIP_C SM_IM SM_CM SM_SD SM_SE SM_GM ATS_IV

ATS_AP ATS_DC ${ }^{-}$ATS_GS ${ }_{-}^{-}$Ship_V ${ }^{-}$Ship_B ${ }_{-}^{-}$MomSES

DadSES;

USEVARIABLES ClassNum GPA Plans SM_IM SM_CM SM_SD

SM_SE SM_GM ATS_IV ATS_AP ATS_DC ATS_GS;

CLASSES $=\mathrm{c}(4)$

! Four latent profiles specified, based on results of original iterative modeling process

AUXILLARY = Sex Ship_V Ship_B MomSES DadSES PersProf; ! Now, covariates are included in the AUXILIARY statement so that they will be included in the !datafile outputted by the SAVEDATA command at the bottom of the syntax file.

\section{MISSING ARE .;}

ANALYSIS:

TYPE=MIXTURE;

STARTS $=2000$ 500;

! Added increased number of starts for each step of the ML estimation in response to message

! about possible convergence issue noted in output

MODEL:

!Want to run the model that was decided upon through the enumeration phase.

!For a default Mplus model the LPA model does not need to be specified. However, it can be. !The model can also be modified from the Mplus default of estimating the indicator means !(uniquely across profiles) and variances (constrained across profiles), as well as the latent !profile mean. The syntax below specifies the Mplus defaults.

$\% O V E R A L L \%$ 
[ClassNum GPA Plans SM_IM SM_CM SM_SD SM_SE SM_GM ATS_IV

ATS_AP ATS_DC ATS_GS]; ! estimates the indicator means for each profile. Without a label !after the brackets, the means are freely estimated in each profile, not constrained.

ClassNum GPA Plans SM_IM SM_CM SM_SD SM_SE SM_GM ATS_IV

ATS_AP ATS_DC ATS_GS (Var1-Var12); !Label Var1-Var12 constrains the estimates of the !variances across the profiles to be equal.

OUTPUT:

TECH1 TECH8 TECH11 TECH 14;

SAVEDATA:

FILE IS LPA1_4_FINAL_Cov.dat;

SAVE = bchweights;

!This statement makes sure that the weights of the indicators for each of the profiles. The bch !weights are based upon the "Classification Probabilities for the Most Likely Latent Class

!Membership (Column) by Latent Class (Row)". These are used in the next modeling step to !specify the profiles so that they are not affected by the inclusion of the covariates in the model. 


\section{Mplus Syntax from Example Study \\ Four-Profile Final Model with BCH Covariate Analysis Syntax \\ !Step 3: Estimating covariate and profile relationships}

TITLE: Latent Profile Analysis Model 1, 4 Profile, Students

DATA:

FILE IS LPA1_4_FINAL_Cov.dat;

!Note: the file name changed to the file outputted from the model run above in Step 2.

VARIABLE:

NAMES ARE ID Sex MomEd MomOcc DadEd

DadOcc ClassNum GPA Plans IPIP N IPIP E IPIP O IPIP A

IPIP_C SM_IM SM_CM SM_SD SM_SE SM_GM $\overline{-}$ ATS_IV

ATS_AP ATS_DC ATS_GS Ship_V ${ }_{-}^{-}$Ship_B - MomSES

DadSES W1-W4 MLC;

!The $\mathrm{BCH}$ weights (W1-W4) are included at the end of the datafile.

USEVARIABLES Sex Ship_V Ship_B MomSES DadSES PersProf W1-W4;

! Now, covariates are included in the USEVARIABLES statement. The BCH weights (W1-W4)

!are also included. Because the $\mathrm{BCH}$ weights are included, the original indicators do not need to !be included in the usevariables. The $\mathrm{BCH}$ weights are unique to the individual, therefore

!retaining the classification uncertainty present in the enumeration step in this model.

CLASSES $=\mathrm{c}(4)$

! Four latent profiles specified, based on results of original iterative modeling process

TRAINING = W1-W4 (bch);

MISSING ARE .;

ANALYSIS:

TYPE=MIXTURE;

STARTS $=0$;

! Note. Starts now 0 because the $\mathrm{BCH}$ weights are specifying the classes based upon the prior

!model run.

MODEL:

$\%$ OVERALL \%

C ON Sex Ship_V Ship_B MomSES DadSES PersProf;

!Latent profiles regressed on the covariate variables. These are uniquely estimated for each class. 
!If there are additional relationships (e.g., PersProf ON Sex) that should be estimated uniquely in !one or more of the classes (i.e., not constrained across classes), then the class-specific syntax !can be added below.

OUTPUT:

TECH1 TECH8 TECH11 TECH 14; 


\section{Appendix D: Mplus Syntax from Example Study}

Four-Profile Final Model with VAM (three-step) Covariate Analysis Syntax

Step 2, after enumeration phase completed is to create a file with the class posterior probabilities along with the covariates by including them as auxiliary variables.

TITLE: Latent Profile Analysis Model 1, 4 Profile, Students

DATA:

FILE IS Mplus_LPA_RQ1_Final.csv;

VARIABLE:

NAMES ARE ID Sex MomEd MomOcc DadEd

DadOcc ClassNum GPA Plans IPIP_N $\quad$ IPIP_E IPIP_O IPIP_A

IPIP_C SM_IM SM_CM SM_SD SM_SE SM_GM ATS_IV

ATS_AP ATS_DC ${ }^{-}$ATS_GS Ship_V Ship_B - MomSES

DadSES;

USEVARIABLES ClassNum GPA Plans SM_IM SM_CM SM_SD

SM_SE SM_GM ATS_IV ATS_AP ATS_DC ATS_GS;

CLASSES $=\mathrm{c}(4)$

! Four latent profiles specified, based on results of original iterative modeling process

AUXILLARY = Sex Ship_V Ship_B MomSES DadSES PersProf; ! Now, covariates are included in the AUXILIARY statement so that they will be included in the !datafile outputted by the SAVEDATA command at the bottom of the syntax file.

MISSING ARE ;

ANALYSIS:

TYPE=MIXTURE;

STARTS $=2000$ 500;

! Added increased number of starts for each step of the ML estimation in response to message

! about possible convergence issue noted in output

MODEL:

!Want to run the model that was decided upon through the enumeration phase.

!For a default Mplus model the LPA model does not need to be specified. However, it can be. !The model can also be modified from the Mplus default of estimating the indicator means !(uniquely across profiles) and variances (constrained across profiles), as well as the latent !profile mean. The syntax below specifies the Mplus defaults.

$\%$ OVERALL $\%$ 
[ClassNum GPA Plans SM_IM SM_CM SM_SD SM_SE SM_GM ATS_IV

ATS_AP ATS_DC ATS_GS]; ! estimates the indicator means for each profile. Without a label !after the brackets, the means are freely estimated in each profile, not constrained.

ClassNum GPA Plans SM_IM SM_CM SM_SD SM_SE SM_GM ATS_IV

ATS_AP ATS_DC ATS_GS (Var1-Var12); !Label Var1-Var12 constrains the estimates of the !variances across the profiles to be equal.

OUTPUT:

TECH1 TECH8 TECH11 TECH 14;

SAVEDATA:

FILE IS LPA1_4_FINAL_Cov_VAM.dat;

SAVE $=$ CPROB;

!This statement makes sure that the class posterior probabilities and most likely classification (MODAL column) are saved along with the variables in the USE and AUXILIARY statements. 


\section{Mplus Syntax from Example Study \\ Four-Profile Final Model with VAM (three-step) Covariate Analysis Syntax \\ !Step 3: Estimating covariate and profile relationships}

TITLE: Latent Profile Analysis Model 1, 4 Profile, Students

DATA:

FILE IS LPA1_4_FINAL_Cov_VAM.dat;

!Note: the file name changed to the file outputted from the model run above in Step 2.

VARIABLE:

NAMES ARE ID Sex MomEd MomOcc DadEd

DadOcc ClassNum GPA Plans IPIP_N IPIP_E IPIP_O IPIP_A

IPIP_C SM_IM SM_CM SM_SD SM_SE SM_GM $\overline{-}$ ATS_IV

ATS_AP ATS_DC ATS_GS Ship_V Ship_B - MomSES

DadSES CPROB1 CPROB2 CPROB3 CPROB4 MODAL;

!The there is a posterior probability for each enumerated class (in this case 4) included at the end of the datafile. The values in these CPROB columns are unique to the individual, so including them increases the

USEVARIABLES Sex Ship_V Ship_B MomSES DadSES PersProf MODAL;

! Now, covariates are included in the USEVARIABLES statement. The MODAL is also !included, because it provides the classification for each individual in the dataset (i.e., class !assignment to class $1,2,3$, or 4 in this example).

NOMINAL ARE MODAL; !Necessary, because the MODAL variable is nominal.

CLASSES $=\mathrm{c}(4)$

! Four latent profiles specified, based on results of original iterative modeling process

MISSING ARE .;

ANALYSIS:

TYPE=MIXTURE;

STARTS $=0$;

! Note. Starts now 0 because the $\mathrm{BCH}$ weights are specifying the classes based upon the prior !model run.

MODEL:

$\% O V E R A L L \%$

C ON Sex Ship_V Ship_B MomSES DadSES PersProf;

!General statement to regression a variable onto the latent profile. 
!Below is how the same regressions can be specified if the researcher wants to label the unique !regressions by class to use the labels later in either MODEL CONSTRAINT or MODEL TEST. !C\#1 ON Sex (reg11);

!C\#1 ON Ship_V (reg12);

!C\#1 ON Ship_B (reg13);

!C\#1 ON MomSES (reg14);

!C\#1 ON DadSES (reg15);

!C\#1 ON PersProf (reg16);

!C\#2 ON Sex (reg21);

!C\#2 ON Ship_V (reg22);

!C\#2 ON Ship_B (reg23);

!C\#2 ON MomSES (reg24);

!C\#2 ON DadSES (reg25);

!C\#2 ON PersProf (reg26);

!C\#3 ON Sex (reg31);

!C\#3 ON Ship_V (reg32);

!C\#3 ON Ship_B (reg33);

!C\#3 ON MomSES (reg34);

!C\#3 ON DadSES (reg35);

!C\#3 ON PersProf (reg36);

!Latent profiles regressed on the covariate variables. These are uniquely estimated for each class. !These statements are included for k-1 classes in the syntax as the last class is the reference class. !If there are additional relationships (e.g., PersProf ON Sex) that should be estimated uniquely in !one or more of the classes (i.e., not constrained across classes) with the rest of the class-specific !syntax below.

$\% \mathrm{C} \# 1 \%$

[MODAL\#1@];

[MODAL\#2@];

[MODAL\#3@];

[Sex] (mean11);

[Ship_V] (mean12);

[Ship_B] (mean13);

[MomSES] (mean14);

[DadSES] (mean15);

[PersProf] (mean16);

Sex;

Ship_V;

Ship_B;

MomSES; 


\author{
DadSES; \\ PersProf; \\ $\% \mathrm{C} \# 2 \%$ \\ [MODAL\#1@]; \\ [MODAL\#2@]; \\ [MODAL\#3@];
}

[Sex] (mean21);

[Ship_V] (mean22);

[Ship_B] (mean23);

[MomSES] (mean24);

[DadSES] (mean25);

[PersProf] (mean26);

Sex;

Ship_V;

Ship_B;

MomSES;

DadSES;

PersProf;

$\% \mathrm{C} \# 3 \%$

[MODAL\#1@];

[MODAL\#2@];

[MODAL\#3@];

[Sex] (mean31);

[Ship_V] (mean32);

[Ship_B] (mean33);

[MomSES] (mean34);

[DadSES] (mean35);

[PersProf] (mean36);

Sex;

Ship_V;

Ship_B;

MomSES;

DadSES;

PersProf;

$\% \mathrm{C} \# 4 \%$

[MODAL\#1@]; 
[MODAL\#2@]; [MODAL\#3@];

[Sex] (mean41);

[Ship_V] (mean42);

[Ship_B] (mean43);

[MomSES] (mean44);

[DadSES] (mean45);

[PersProf] (mean46);

Sex;

Ship_V;

Ship_B;

MomSES;

DadSES;

PersProf;

!The means and variances are uniquely estimated in each class for each variable of interest. If !there were specific relationships to model between any of these variables, the regressions would !be included in the class-specific syntax. Unique labels can be used in MODEL CONSTRAINT !and/or MODEL TEST commands as is done with other model types to test class differences and !indirect effects.

MODEL TEST:

mean $11=$ mean 21

mean $11=$ mean 31

mean $11=$ mean 41

mean $21=$ mean 31

mean $21=\operatorname{mean} 41$

mean $31=$ mean 41

mean $12=$ mean 22 ;

mean $12=$ mean 32 ;

mean $12=$ mean 42 ;

mean $22=$ mean $32 ;$

mean $22=$ mean $42 ;$

mean $32=$ mean 42 ;

mean $13=$ mean 23 ;

mean13 = mean33;

mean $13=$ mean 43 ;

mean $23=$ mean 33 ;

mean $23=$ mean 43 ;

mean33 = mean43;

mean14 = mean24; 


$$
\begin{aligned}
& \text { mean14 = mean34; } \\
& \text { mean } 14=\text { mean } 44 \text {; } \\
& \text { mean24 = mean } 34 \text {; } \\
& \text { mean } 24=\text { mean } 44 ; \\
& \text { mean34 = mean44; } \\
& \text { mean } 15=\text { mean } 25 \text {; } \\
& \text { mean } 15=\text { mean } 35 \text {; } \\
& \text { mean } 15=\text { mean } 45 \\
& \text { mean } 25=\text { mean } 35 \text {; } \\
& \text { mean25 = mean } 45 \text {; } \\
& \text { mean } 35=\text { mean } 45 \text {; } \\
& \text { mean } 16=\text { mean } 26 \text {; } \\
& \text { mean } 16=\text { mean } 36 \text {; } \\
& \text { mean } 16=\text { mean } 46 \text {; } \\
& \text { mean } 26=\text { mean } 36 \text {; } \\
& \text { mean26 }=\text { mean } 46 \text {; } \\
& \text { mean36 }=\text { mean46; }
\end{aligned}
$$

!The above syntax is directly testing the means of the variables in the different classes

OUTPUT:

\section{TECH1 TECH8 TECH11 TECH 14;}

